\title{
B-Spline Collocation Method for Solving Singularly Perturbed Boundary Value Problems
}

\author{
Bin Lin \\ School of Mathematics and Computation Science, Lingnan Normal University, Zhanjiang, China \\ Email: linbin167@163.com, linbin@stu.xjtu.edu.cn
}

How to cite this paper: Lin, B. (2016) B-Spline Collocation Method for Solving Singularly Perturbed Boundary Value Problems. Journal of Applied Mathematics and Physics, 4, 1699-1704.

http://dx.doi.org/10.4236/jamp.2016.49178

Received: July 18, 2016

Accepted: September 4, 2016

Published: September 7, 2016

Copyright $\odot 2016$ by author and Scientific Research Publishing Inc. This work is licensed under the Creative Commons Attribution International License (CC BY 4.0).

http://creativecommons.org/licenses/by/4.0/

\begin{abstract}
We use fifth order B-spline functions to construct the numerical method for solving singularly perturbed boundary value problems. We use B-spline collocation method, which leads to a tri-diagonal linear system. The accuracy of the proposed method is demonstrated by test problems. The numerical results are found in good agreement with exact solutions.
\end{abstract}

\section{Keywords}

Fifth Order B-Spline Functions, B-Spline Collocation Method, Singularly Perturbed Boundary Value Problems

\section{Introduction}

Consider following singularly perturbed boundary value problem

$$
L y(x)=-\varepsilon y^{\prime \prime}+p(x) y^{\prime}+q(x) y=f(x), a<x<b
$$

with boundary conditions

$$
y(a)=A, \quad y(b)=B, \quad y^{\prime}(a)=\gamma, \quad y^{\prime}(b)=\delta
$$

where $0<\varepsilon<1, \varepsilon$ is a small positive parameter, $p(x)$ and $q(x)$ are sufficiently smooth real-valued functions. Typically, these problems arise very frequently in fluid dynamics, elasticity, quantum mechanics, chemical reactor theory and many other allied areas. Up to now, different numerical methods have been proposed by various authors [1]-[3] for this singularly perturbed problem arising in transport phenomena in chemistry and biology [4]. It is so attractive to mathematicians due to the fact that the solution exhibits a multi-scale character, i.e., there is a thin layer where the solution varies rapidly, while away from the layer the solution behaves regularly and varies slowly. So the usual numerical treatment of singular perturbation problems gives rise to major 
computational difficulties and fails to give accurate solutions.

B-spline functions are useful wavelet basis functions; the stiffness matrix is sparse when it is used as trial functions. B-splines were introduced by Schoenberg in 1946 [5]. Up to now, B-spline approximation method for numerical solutions has been researched by various researchers [6]-[8].

\section{Description of the B-Spline Collocation Method}

The expression of fifth order B-spline function is as follows:

$$
N_{6}(x)= \begin{cases}(x+3)^{5} & {[-3,-2)} \\ (x+3)^{5}-6(x+2)^{5} & {[-2,-1)} \\ (x+3)^{5}-6(x+2)^{5}+15(x+1)^{5} & {[-1,0)} \\ (3-x))^{5}-6(2-x)^{5}+15(1-x)^{5} & {[0,1)} \\ (3-x))^{5}-6(2-x)^{5} & {[1,2)} \\ (3-x)^{5} & {[2,3)} \\ 0 & \text { others }\end{cases}
$$

The fifth order B-spline function $N_{6}(x)$ is used to calculate in this work and possesses the following characters: piecewise smooth, compact support, Symmetry, rapidly decaying, differentiability, linear combination.

The region $[a, b]$ is partitioned into uniformly sized finite elements of length $h$ by the knots $x_{j}$ such that $a=x_{0}<x_{1}<x_{2}<\cdots<x_{N}=b$ with $h=x_{j}-x_{j-1}, x_{j}=a+j h$, $j=1,2, \cdots, N$. Let $\phi_{m}(x)$ be fifth order B-spline function with knots at the points $x_{m}$, $m=0,1, \cdots, N$. The set of splines $\left\{\phi_{-2}, \phi_{-1}, \phi_{0}, \phi_{1}, \cdots, \phi_{N}, \phi_{N+1}, \phi_{N+2}\right\}$ forms a basis for functions defined over $[a, b]$.

In the proposed algorithm, The fifth order B-spline function $N_{6}(x)$ is used as a single mother wavelet, i.e. $\phi(x)=N_{6}(x)$ and dilation and translation of mother wavelet functions can construct any function of $L^{2}(R)$.

$$
S(x)=\sum_{k} c_{k} \phi_{k}^{J}(x)=\sum_{k} c_{k} 2^{J / 2} \phi\left(2^{J} x-k\right)=\sum_{k} a_{k} \phi\left(\frac{x-2^{-J} k}{2^{-J}}\right)=\sum_{k} a_{k} N_{6}\left(\frac{x-x_{k}}{h}\right)
$$

where $h=1 / 2^{J}, x_{k}=k / 2^{J}, a_{k}=2^{J / 2} c_{k}$.

So the global approximation $S(x)$ to the function $y(x)$ can be written in terms of the B-spline as follows

$$
S(x)=\sum_{i=-2}^{N+2} a_{i} N_{6}\left(\frac{x-x_{i}}{h}\right)
$$

where $h=\frac{b-a}{n}, a_{i}$ are unknown real coefficients.

Using the fifth order B-spline function and the approximate solution Equation (5), the nodal values $S\left(x_{j}\right), S^{\prime}\left(x_{j}\right)$ and $S^{\prime \prime}\left(x_{j}\right)$ at the node $x_{j}$ are given in terms of element parameters by 


$$
\begin{gathered}
S\left(x_{j}\right)=\frac{1}{120}\left(a_{j-2}+26 a_{j-1}+66 a_{j}+26 a_{j+1}+a_{j+2}\right) \\
S^{\prime}\left(x_{j}\right)=\frac{1}{24 h}\left(-a_{j-2}-10 a_{j-1}+10 a_{j+1}+a_{j+2}\right) \\
S^{\prime \prime}\left(x_{j}\right)=\frac{1}{6 h^{2}}\left(a_{j-2}+2 a_{j-1}-6 a_{j}+2 a_{j+1}+a_{j+2}\right)
\end{gathered}
$$

where the symbols ' and " denote first and second differentiation with respect to $X$, respectively.

Substituting Equations (6)-(8) into Equation (1) and Equation (2), we can obtain following linear equations

$$
B a=r
$$

where

$$
\begin{gathered}
r=\left(120 y(a), 24 h y^{\prime}(a),-120 h^{2} f_{0} / \varepsilon, \cdots,-120 h^{2} f_{N} / \varepsilon, 24 h y^{\prime}(b), 120 y(b)\right)^{\mathrm{T}} \\
a=\left(a_{-2}, a_{-1}, a_{0}, a_{1}, \cdots, a_{N}, a_{N+1}, a_{N+2}\right)^{\mathrm{T}}, \quad f_{i}=f(a+i h)
\end{gathered}
$$

Note $N_{6}\left(\frac{x_{j}-x_{i}}{h}\right)=B_{i j}$

$$
B=\left[\begin{array}{ccccccc}
1 & 26 & 66 & 26 & 1 & 0 & \\
-1 & -10 & 0 & 10 & 1 & 0 & \\
L B_{-2,0} & L B_{-1,0} & L B_{0,0} & L B_{1,0} & L B_{2,0} & & \\
& \ddots & \ddots & \ddots & \ddots & \ddots & \\
& & L B_{N-2, N} & L B_{N-1, N} & L B_{N, N} & L B_{N+1, N} & L B_{N+2, N} \\
& & -1 & -10 & 0 & 10 & 1 \\
& & 1 & 26 & 66 & 26 & 1
\end{array}\right]
$$

where

$$
\begin{aligned}
& L B_{j-2, j}=20+5 h p_{j} / \varepsilon-h^{2} q_{j} / \varepsilon, L B_{j-1, j}=40+50 h p_{j} / \varepsilon-26 h^{2} q_{j} / \varepsilon \\
& L B_{j, j}=-120-66 h^{2} q_{j} / \varepsilon, \quad L B_{j+1, j}=40-50 h p_{j} / \varepsilon-26 h^{2} q_{j} / \varepsilon \\
& L B_{j+2, j}=20-5 h p_{j} / \varepsilon-h^{2} q_{j} / \varepsilon, \quad p_{j}=p(a+j h), \quad q_{j}=q(a+j h)
\end{aligned}
$$

It is easily seen that the matrix $B$ is strictly diagonally dominant and hence nonsingular. Since $B$ is nonsingular, we can solve the system $B a=r$ for

$a_{-2}, a_{-1}, a_{0}, a_{1}, \cdots, a_{N}, a_{N+1}, a_{N+2}$. Hence the method of collocation using the fifth order B-spline function $N_{6}(x)$ as a basis function applied to the singularly perturbed boundary value problem has a unique solution $S(x)$ given by Equation (5).

\section{Numerical Results}

In the section, we illustrate the numerical techniques discussed in the previous section by the following problems.

Example 1. Consider the convention-dominated equation:

$$
-\varepsilon y^{\prime \prime}+y^{\prime}+y=1 \quad(0<x<1)
$$


with boundary conditions: $y(0)=y(1)=0$,

$$
\begin{gathered}
y^{\prime}(0)=\left[\lambda_{1}\left(\mathrm{e}^{\lambda_{2}}-1\right)+\lambda_{2}\left(1-\mathrm{e}^{\lambda_{1}}\right)\right] /\left(\mathrm{e}^{\lambda_{1}}-\mathrm{e}^{\lambda_{2}}\right), \\
y^{\prime}(1)=\left[\lambda_{1}\left(\mathrm{e}^{\lambda_{2}}-1\right) \mathrm{e}^{\lambda_{1}}+\lambda_{2}\left(1-\mathrm{e}^{\lambda_{1}}\right) \mathrm{e}^{\lambda_{2}}\right] /\left(\mathrm{e}^{\lambda_{1}}-\mathrm{e}^{\lambda_{2}}\right) .
\end{gathered}
$$

The exact solution is given by

$$
y(x)=\left(\mathrm{e}^{\lambda_{2}}-1\right) \mathrm{e}^{\lambda_{1} x} /\left(\mathrm{e}^{\lambda_{1}}-\mathrm{e}^{\lambda_{2}}\right)+\left(1-\mathrm{e}^{\lambda_{1}}\right) \mathrm{e}^{\lambda_{2} x} /\left(\mathrm{e}^{\lambda_{1}}-\mathrm{e}^{\lambda_{2}}\right)+1
$$

where $\lambda_{1}=(1+\sqrt{1+4 \varepsilon}) /(2 \varepsilon), \lambda_{2}=(1-\sqrt{1+4 \varepsilon}) /(2 \varepsilon)$.

Comparison of the numerical results and point-wise errors is given in Table 1.

It observed that

1) when $h$ decreases (i.e. collocation number increases) for fixed $\varepsilon$ the point-wise errors decrease;

2) when $\varepsilon$ decreases for fixed $h$ the point-wise errors increase;

3) when $\varepsilon=0.0015, x \rightarrow 1$ the errors are very large.

Example 2. Solve the following non-homogeneous equation:

$$
-\varepsilon y^{\prime \prime}+p y^{\prime}+y=\cos \pi x \quad(0<x<1)
$$

with boundary conditions

$$
y(0)=y(1)=0, \quad y^{\prime}(0)=b \pi+A \lambda_{1}+\lambda_{2} B \mathrm{e}^{-\lambda_{2}}, \quad y^{\prime}(1)=-b \pi+A \lambda_{1} \mathrm{e}^{\lambda_{1}}+\lambda_{2} B .
$$

The analytical solution is given by

$$
y(x)=a \cos \pi x+b \sin \pi x+A \exp \left(\lambda_{1} x\right)+B \exp \left[-\lambda_{2}(1-x)\right]
$$

where

$$
a=\frac{\varepsilon \pi^{2}+1}{p^{2} \pi^{2}+\left(\varepsilon \pi^{2}+1\right)^{2}}, b=\frac{p \pi}{p^{2} \pi^{2}+\left(\varepsilon \pi^{2}+1\right)^{2}}, \quad A=-a \frac{1+\exp \left(-\lambda_{2}\right)}{1-\exp \left(\lambda_{1}-\lambda_{2}\right)}, B=a \frac{1+\exp \left(\lambda_{1}\right)}{1-\exp \left(\lambda_{1}-\lambda_{2}\right)} .
$$

\begin{tabular}{|c|c|c|c|c|c|c|c|c|c|}
\hline \multirow{2}{*}{$\mathrm{X}$} & \multirow{2}{*}{$\begin{array}{c}\varepsilon=0.1 \\
h=1 / 32 \\
\text { error }\end{array}$} & \multicolumn{3}{|c|}{$\varepsilon=0.1, h=1 / 128$} & \multirow{2}{*}{$\begin{array}{c}\varepsilon=0.01 \\
h=1 / 32 \\
\text { error }\end{array}$} & \multicolumn{3}{|c|}{$\varepsilon=0.01, h=1 / 128$} & \multirow{2}{*}{$\begin{array}{c}\varepsilon=0.0015 \\
h=1 / 1024 \\
\text { error }\end{array}$} \\
\hline & & numerical & Exact & error & & Numerical & Exact & error & \\
\hline $2 / 16$ & 0.0037 & 0.1090 & 0.1082 & 0.0008 & 0.0092 & 0.1176 & 0.1164 & 0.0012 & 0.0001465 \\
\hline $4 / 16$ & 0.0033 & 0.2053 & 0.2045 & 0.0007 & 0.0082 & 0.2204 & 0.2193 & 0.0011 & 0.0001294 \\
\hline $12 / 16$ & 0.0021 & 0.0004 & 0.4582 & 0.4578 & 0.0052 & 0.5248 & 0.5241 & 0.0007 & 0.0000785 \\
\hline $14 / 16$ & 0.0014 & 0.3984 & 0.3981 & 0.0003 & 0.0025 & 0.5801 & 0.5795 & 0.0006 & 0.0000693 \\
\hline 1 & 0.0004 & 0.0462 & 0.0462 & 0 & 0.0199 & 0.3402 & 0.3401 & 0.0001 & 0.0107 \\
\hline
\end{tabular}

And $\lambda_{1}<0$ and $\lambda_{2}>0$ are the real solutions of the characteristic equation $-\varepsilon \lambda^{2}+p \lambda+1=0$.

Approximation solutions for different values of $\varepsilon$ and for fixed $p$ are given in Figure 1. It observed that

Table 1. Example 1. Comparison of results and point-wise errors. 
approximate solutions of example 2. for different values of epsiling and for fix $\mathrm{p}$

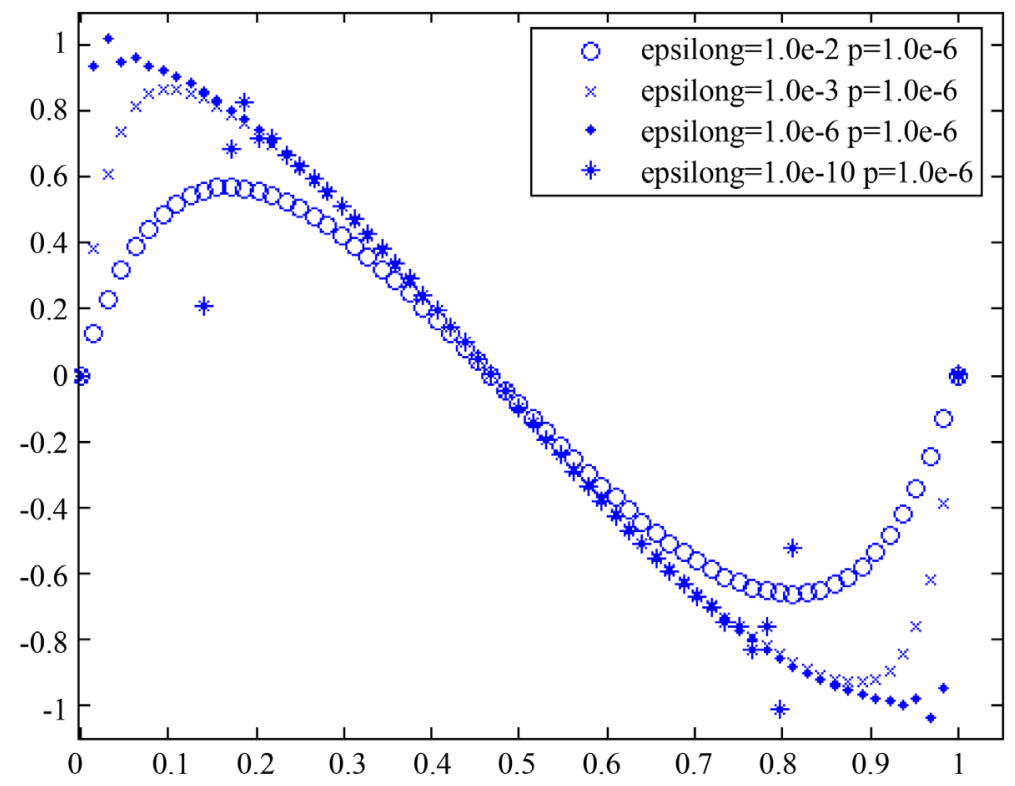

Figure 1. Approximation solutions of example 2 for different values of epsilon $g$ and for fixed $\mathrm{p}$.

1) when $\varepsilon=10^{-2}$ and $10^{-3}$, the approximation solutions are in good agreement with exact solution; 2) when $\varepsilon=10^{-6}$ and $10^{-10}, x \rightarrow 0$ and $x \rightarrow 1$ the errors are very large; 3 ) when $\varepsilon$ decreases for fixed $p$ the width of boundary layer becomes small and wave shape change more and more stiff at $x=0$ and $x=1$.

\section{Conclusion}

The numerical results show clearly the effect of $\varepsilon$ on the boundary layer and the B-spline collocation method solving singular boundary value problems is relatively simple to collocate the solution at the mesh points. It is applicable technique and approximates the exact solution very well.

\section{Acknowledgements}

The authors would like to thank the editor and the reviewers for their valuable comments and suggestions to improve the results of this paper. This work was supported by the Natural Science Foundation of Guangdong (No. 2015A030313827).

\section{References}

[1] Evrenosoglu, M. and Somali, S. (2008) Least Squares Methods for Solving Singularly Perturbed Two-Point Boundary Value Problems Using Bezier Control Points. Applied Mathematics Letters, 21, 1029-1032. http://dx.doi.org/10.1016/j.aml.2007.10.021

[2] Lin, B., Li, K.T. and Cheng, Z.X. (2009) B-Spline Solution of a Singularly Perturbed Boundary Value Problem Arising in Biology. Chaos, Solitons \& Fractals, 42, 2934-2948. http://dx.doi.org/10.1016/j.chaos.2009.04.036

[3] Fan, X.H. and Tian, L.X. (2005) The Existence of Solitary Waves of Singularly Perturbed 
mKdV-KS Equation. Chaos, Solitons \& Fractals, 26, 1111-1118.

http://dx.doi.org/10.1016/j.chaos.2005.02.014

[4] Bigge, J. and Bohl, E. (1985) Deformations of the Bifurcation Diagram Due to Discretization. Mathematics of Computation, 45, 393-403.

http://dx.doi.org/10.1090/S0025-5718-1985-0804931-X

[5] De Boor, C. (1978) A Practical Guide to Splines. Springer-Verlag, Berlin. http://dx.doi.org/10.1007/978-1-4612-6333-3

[6] Siddiqi, S.S. and Akram, G. (2007) Sextic Spline Solutions of Fifth Order Boundary Value Problems. Applied Mathematics Letters, 20, 591-597.

http://dx.doi.org/10.1016/j.aml.2006.06.012

[7] Caglar, H.N. and Caglar, S.H. (1997) The Numerical Solution of Fifth Order Boundary Value Problems with Sixth Degree B-Spline Functions. Applied Mathematics Letters, 12, 25-30. http://dx.doi.org/10.1016/S0893-9659(99)00052-X

[8] Cağlar, H., Özer, M. and Cağlar, N. (2008) The Numerical Solution of the One-Dimensional Heat Equation by Using Third Degree B-Spline Functions. Chaos, Solitons \& Fractals, 38, 1197-1201. http://dx.doi.org/10.1016/j.chaos.2007.01.056

Submit or recommend next manuscript to SCIRP and we will provide best service for you:

Accepting pre-submission inquiries through Email, Facebook, LinkedIn, Twitter, etc. A wide selection of journals (inclusive of 9 subjects, more than 200 journals)

Providing 24-hour high-quality service

User-friendly online submission system Fair and swift peer-review system

Efficient typesetting and proofreading procedure

Display of the result of downloads and visits, as well as the number of cited articles Maximum dissemination of your research work

Submit your manuscript at: http://papersubmission.scirp.org/ 